\title{
Materialize Secluded Data Integrity Checking Protocol For Secured Storage Services With Data Dynamics And Public Verifiability In Hybrid Cloud
}

\author{
Ms. Vaishali Dubey, Dr. A. Gururani \\ Department Of Computer Science \& Engineering Dayananda Sagar College Of \\ Engineering, Bangalore, India
}

\begin{abstract}
Data desire to stress concerning the technical implementations and nitty-gritties like the software's physical hosting location, hardware specifications, potency of knowledge process. Recently several works specialize in providing data Dynamics and/or public verifiability to the protocols checking the secluded integrity with the assistance of third party verifiers. during this paper, Seb'e et al.'s protocol is customized to support public verifiability and data Dynamics while not the assistance of a 3rd party auditor. This style permits users to audit the cloud storage with terribly light-weight communication and computation value. additionally, the auditing result not solely ensures robust cloud storage correctness guarantee, however additionally at the same time achieves quick data error localization, i.e., the identification of misbehaving server. the planning more supports secure and economical dynamic operations on outsourced information, as well as block modification, deletion, and append. Through a proper analysis, the correctness and security of the protocol is shown. The planned theme is extremely economical and resilient against the malicious data modification attack, server evaporation attacks and failure.
\end{abstract}

Keyword - Cloud computing, data dynamics, data integrity, error localization, public verifiability, Seb'e'etal's protocol.

\section{Introduction}

Hybrid cloud is that the long unreal vision of computing as a utility, wherever users will remotely store their data into the cloud thus on fancy prime quality applications and services from a shared pool of configurable computing resources (fig 1). it's been visualised because the on-demand selfservice, present network access, location freelance resource pooling, fast resource physical property, usage-based rating and transference of risk [1]. It works on a client-server basis, 
exploitation browser protocols. A cloud user wants a consumer device like a laptop computer or PC, pad laptop, sensible phone, or different computing resource with an online browser (or different approved access route) to access a cloud system via the globe Wide internet. usually the user can log into the cloud at a service supplier or non-public company, like their leader. The cloud provides server-based applications and every one data services to the user, with output displayed on the consumer device. Memory allotted to the consumer system's browser is employed to create the appliance data seem on the consumer system show, however all computations and changes area unit recorded by the server, and final results as well as files created or altered area unit for good keep on the cloud servers. Performance of the cloud application depends upon the network access, speed and responsibleness also because the process speed of the consumer device.

As a result, the correctness of the info within the cloud is being place in danger thanks to the subsequent reasons. initial of all, though the infrastructures underneath the cloud area unit way more powerful and reliable than personal computing devices, they're still facing the broad vary of each internal and external threats for data integrity [3][4][5]. Secondly, for the advantages of their own, there do exist varied motivations for cloud service suppliers to behave unreliably towards the cloud users relating to the standing of their outsourced data[6][7]. These issues, impedes the victorious preparation of the cloud design. The increasing network data measure and reliable nonetheless versatile network connections build it even attainable that users will currently subscribe prime quality services from data and package that reside entirely on secluded data centres

In this paper, the most contributions of the planned Seb'e et al.'s protocol are:

(1) a far off data integrity checking protocol for cloud storage, which might be viewed as associate adaptation of Seb'e et al.'s protocol [14]. The planned protocol inherits the support of knowledge dynamics from and supports public verifiability and privacy against third party verifiers, whereas at an equivalent time it doesn't got to use a third-party auditor.

(2) A security analysis of the planned protocol, that shows that it's secure against the untrusted server and personal against third party verifiers, is given [14]. 


\section{THE AMERICAN JOURNAL OF}

ENGINEERING AND TECHNOLOGY

(3) Compared to several of its predecessors, that solely offer binary results concerning the storage standing across the distributed servers, the planned theme achieves the combination of storage correctness insurance and data error localization, i.e., the identification of misbehaving server(s).

\section{Background Work}

2.1 Existing System within the existing system cloud data storage service involve 3 completely different entities, the cloud user (U), UN agency has great amount of knowledge files to be keep within the cloud; the cloud server (CS), that is managed by cloud service supplier (CSP) to produce data storage service; the third party auditor (TPA), UN agency has experience and capabilities that cloud users don't have and is trusty to assess the cloud storage service security on behalf of the user upon request. Users think about the metal for cloud data storage and maintenance. The TPA, UN agency is within the business of auditing, is assumed to be reliable and freelance, and so has no incentive to interact with either the metal or the users throughout the auditing method.

\section{Planned System}

In planned system a good and versatile distributed theme with specific dynamic data support, as well as block update, delete, and append. the variation of planned protocol- Seb'e et al.'s protocol with distributed verification of erasure-coded information, the theme achieves the general public verifiability and data Dynamics against the third party verifiers that shows the detection of knowledge corruption throughout the storage correctness verification across the distributed servers .

Identification Chance For Misbehaving Servers

Ms. Vaishali Dubey, Dr. A. Gururani 
From user's perspective, the model has got to capture all types of threats towards the cloud data integrity. as a result of cloud data don't reside at user's native web site however at CSP's address domain, these threats will come back from 2 completely different sources: internal and external attacks. For internal attacks, the domain will be self-interested, untrusted and probably malicious. Not solely will it want to maneuver data that has not been or is never accessed to a lower tier of storage than united for financial reasons, however it's going to additionally arrange to hide a knowledge loss incident thanks to management errors. For external attacks, data integrity threats might come back from outsiders UN agency area unit on the far side the management domain, for instance, the economically impelled attackers. they will compromise variety of cloud data storage servers in several time intervals and after be ready to modify or delete users' data whereas remaining undetected. Therefore, the planned model has the capabilities, that captures each external and internal threats towards the cloud data integrity. Suppose $n$ servers area unit misbehaving thanks to the attainable compromise failure, assume the mortal modifies the info blocks in z rows out of the I rows within the encoded file matrix. Let $r$ be the quantity of various rows that the user asks for checking in a very challenge. Let $X$ be $a$ distinct variable that's outlined to be the quantity of rows chosen by the user that matches the rows changed by the mortal.

\section{Related Work}

Shah of Iran et al. [8], [9] propose permitting a TPA to stay on-line storage honest by initial encrypting \{the information/the info|the information\} then causing variety of pre-computed symmetric-keyed hashes over the encrypted data to the auditor. The auditor verifies each the integrity of the info file and also the server's possession of a antecedently committed cryptography key. This theme solely works for encrypted files and it suffers from the auditor statefulness and delimited usage, which can probably herald on-line burden to users once the keyed hashes area unit wiped out. Ateniese et al. [6] were the primary UN agency outlined the "provable data possession" (PDP) model for guaranteeing possession of file on untrusted storages. . Their theme utilizes the RSA-based homomorphic authenticators for auditing outsourced data and suggests willy-nilly sampling a couple of blocks of the file. However, the general public auditability in their theme demands the linear combination of sampled blocks exposed to external auditor. once used directly, their protocol isn't demonstrably privacy protective, and so might leak user data info to the auditor. In their resulting work, Ateniese et al. [10] delineated a PDP theme that uses solely bilaterally symmetric key based mostly cryptography. This technique has lower-overhead than their previous theme and permits for 
block updates, deletions and appends to the keep file, that has additionally been supported in our work.

\section{Conclusion \& Future}

add this paper, a privacy-preserving protocol for data storage within the cloud is been planned work the info security and integrity in cloud storage. the main focus is on stopping data being disclosed by un-trusted service suppliers once data homeowners distribute their info entries. to attain the assurances of cloud data integrity and availableness and enforce the standard of dependable cloud storage service for users, a good and versatile distributed theme with specific dynamic data support, as well as block update, delete and append is being enforced. the longer term analysis aims to increase the protocol to support data level dynamics at borderline prices. This includes the in depth security and resilient to the failures like Byzantine failure and different malicious data attacks.

\section{References}

1. T. Grance, "Draft NIST operating definition of cloud computing," documented on June. 3rd, 2007. on-line, 2007.

2. Amazon.com, "Amazon s3 availableness event: Gregorian calendar month twenty, 2008," on-line,2008.

3. S.Wilson,“Application engine outage/711/ appengine outage.php, June 2006.

4. V.Krebs, "Payment Processor Breach is also Largest Ever,"/securityfix/2007/01/payment processor breach might b.html, Jan. 2009.

5. D. Ateniese, R. Burns, R. Curtmola, J. Herring, L. Kissner, Z. Peterson, and D. Song, "Provable datapossession at untrusted stores," scientific discipline ePrint Archive, Report 2006. 
6. C. Wang, J. Li, K. Ren, and W. Lou, "Enabling public verifiability and data Dynamics for storage security. 